\title{
Electron-Pair Densities with Time-Dependent Quantum Monte Carlo
}

\author{
Ivan P. Christov \\ Physics Department, Sofia University, 1164 Sofia, Bulgaria \\ Correspondence should be addressed to Ivan P. Christov; ipc@phys.uni-sofia.bg
}

Received 4 April 2013; Revised 5 June 2013; Accepted 8 July 2013

Academic Editor: Keli Han

Copyright (C) 2013 Ivan P. Christov. This is an open access article distributed under the Creative Commons Attribution License, which permits unrestricted use, distribution, and reproduction in any medium, provided the original work is properly cited.

\begin{abstract}
We use sets of de Broglie-Bohm trajectories to describe the quantum correlation effects which take place between the electrons in helium atom due to exchange and Coulomb interactions. A short-range screening of the Coulomb potential is used to modify the repulsion between the same spin electrons in physical space in order to comply with Pauli's exclusion principle. By calculating the electron-pair density for orthohelium, we found that the shape of the exchange hole can be controlled uniquely by a simple screening parameter. For parahelium the interelectronic distance, hence the Coulomb hole, results from the combined action of the Coulomb repulsion and the nonlocal quantum correlations. In this way, a robust and self-interaction-free approach is presented to find both the ground state and the time evolution of nonrelativistic quantum systems.
\end{abstract}

\section{Introduction}

The electronic many-body problem is of key importance for the theoretical treatments of physics and chemistry. A typical manifestation of the quantum many-body effects is the electron correlation which results from the Coulomb and exchange interactions between the electrons combined with the underlying quantum nonlocality. Since in general the electron correlation reshapes the probability density in configuration space, it is difficult to elucidate this effect for higher dimensions. Therefore, to better understand the effects of electron correlation in atoms and molecules, one needs, besides one-particle quantities such as the electron density function, to consider also extensions which explicitly incorporate many-body effects. Such an appropriate quantity is the electronic pair-density function which represents the probability density of finding two electrons at distance $\mathbf{u}$ from each other $[1,2]$ :

$$
I(\mathbf{u}, t)=\left\langle\Psi(\mathbf{R}, t)\left|\sum_{i<j} \delta\left[\left(\mathbf{r}_{i}-\mathbf{r}_{j}\right)-\mathbf{u}\right]\right| \Psi(\mathbf{R}, t)\right\rangle,
$$

where $\mathbf{r}_{i}$ is the position of the $i$ th electron and the manybody wave function $\Psi(\mathbf{R}, t)$ resides in configuration space with arguments being the instantaneous coordinates of all electrons $\mathbf{R}=\left(\mathbf{r}_{1}, \mathbf{r}_{2}, \ldots, \mathbf{r}_{N}\right)$.

The importance of the electron-pair density, also known as electron position intracule, comes from the fact that it can be associated with experimental data obtained from $\mathrm{X}$-ray scattering, and it can also be used to visualize the notion of exchange and correlation holes which surround the quantum particles. However, the calculation of the manybody wave function in (1) is hampered by the computational cost which scales exponentially with system dimensionality. Therefore, different approximations have been employed in order to calculate the electronic pair densities. These include Hartree-Fock (HF) approximation as well as Hylleraas type explicitly correlated wave functions represented as product of $\mathrm{HF}$ function and pair-correlation factors [3-6]. Other (e.g., quantum Monte Carlo [7]) approaches use appropriate Slater-Jastrow-type many-body wave functions which involve number of parameters, which after optimization can be used to calculate the average in (1).

Here we calculate the electron-pair densities for helium atom in $2{ }^{1} \mathrm{~S}$ and $2{ }^{3} \mathrm{~S}$ states using the recently proposed timedependent quantum Monte Carlo (TDQMC) method which employs sets of particles and quantum waves to describe the ground state and the time evolution of many-electron systems [8-13]. In TDQMC each electron is described statistically 
as an ensemble of walkers which represent different replicas of that electron in position space, where each walker is guided by a separate time-dependent de Broglie-Bohm pilot wave. The correlated guiding waves obey a set of coupled time-dependent Schrödinger equations (TDSE), where the electron-electron interactions are accounted for using explicit nonlocal Coulomb potentials. In the TDQMC algorithm the preparation of the ground state of the quantum system involves a few steps which include initialization of the Monte Carlo (MC) ensembles of walkers and guide waves, followed by their concurrent propagation in complex time toward steady state in the presence of random component in walker's motion to account for the processes of quantum drift and diffusion. Once the ground state is established, the realtime quantum dynamics can be studied, for example, the interaction of atoms and molecules with external electromagnetic fields. The large speedup of the calculations when using TDQMC comes from the fact that walker's distribution reproduces the amplitude (or modulus square) of the many-body wave function, while its phase is being disregarded as it is not needed for most applications. Also, the TDQMC method can be implemented very efficiently on parallel computers where tens of thousands of coupled Schrödinger equations can be solved concurrently for affordable time.

\section{General Theory}

The TDQMC is an $a b$ initio method with respect to the electron correlation in that it does not involve explicit paircorrelation factors which may become too complex when used for larger systems. For a system of $N$ electrons, the many-body wave function obeys the Schrödinger equation:

$$
i \hbar \frac{\partial}{\partial t} \Psi(\mathbf{R}, t)=-\frac{\hbar^{2}}{2 m} \nabla^{2} \Psi(\mathbf{R}, t)+V(\mathbf{R}) \Psi(\mathbf{R}, t),
$$

where $\nabla=\left(\nabla_{1}, \nabla_{2}, \ldots, \nabla_{N}\right)$. The potential $V(\mathbf{R})$ in $(2)$ is a sum of electron-nuclear, electron-electron, and external potentials:

$$
\begin{aligned}
V\left(\mathbf{r}_{1}, \ldots, \mathbf{r}_{N}\right)= & V_{e-n}\left(\mathbf{r}_{1}, \ldots, \mathbf{r}_{N}\right)+V_{e-e}\left(\mathbf{r}_{1}, \ldots, \mathbf{r}_{N}\right) \\
& +V_{\text {ext }}\left(\mathbf{r}_{1}, \ldots, \mathbf{r}_{N}, t\right)=\sum_{k=1}^{N} V_{e-n}\left(\mathbf{r}_{k}\right) \\
& +\sum_{k>l}^{N} V_{e-e}\left(\mathbf{r}_{k}-\mathbf{r}_{l}\right)+V_{\text {ext }}\left(\mathbf{r}_{1}, \ldots, \mathbf{r}_{N}, t\right) .
\end{aligned}
$$

For Hamiltonians with no explicit spin variables the exchange effects can be accounted for efficiently using screened Coulomb potentials as described in [10]. The simple idea behind this approach is that the short-range screened Coulomb potential ensures full-scale Coulomb interaction between only electron replicas (MC walkers) which are not too close to each other, in accordance with Pauli's exclusion principle. The use of screened Coulomb potentials is beneficial in that it eliminates the need of using antisymmetrized products of guiding waves in the Broglie-Bohm guiding equation for the velocity of the walkers. Instead, the manybody wave function is replaced by a simple product:

$$
\Psi^{k}\left(\mathbf{r}_{1}, \mathbf{r}_{2}, \ldots, \mathbf{r}_{N}, t\right)=\prod_{i=1}^{N} \varphi_{i}^{k}\left(\mathbf{r}_{i}, t\right)
$$

where $\varphi_{i}^{k}\left(\mathbf{r}_{i}, t\right)$ denote the individual time-dependent guide waves with indexes $i$ and $k$ for the electrons and the walkers, respectively. Then, the guiding equations for the Monte Carlo walkers read

$$
\mathbf{v}\left(\mathbf{r}_{i}^{k}\right)=\frac{\hbar}{m} \operatorname{Im}\left[\frac{1}{\varphi_{i}^{k}\left(\mathbf{r}_{i}, t\right)} \nabla_{i} \varphi_{i}^{k}\left(\mathbf{r}_{i}, t\right)\right]_{\mathbf{r}_{i}=\mathbf{r}_{i}^{k}(t)} .
$$

On the other side, the guide waves obey a set of coupled TDSE:

$$
\begin{aligned}
i \hbar \frac{\partial}{\partial t} \varphi_{i}^{k}\left(\mathbf{r}_{i}, t\right)=[ & -\frac{\hbar^{2}}{2 m} \nabla_{i}^{2}+V_{e-n}\left(\mathbf{r}_{i}\right) \\
& +\sum_{j \neq i}^{N} V_{e-e}^{\mathrm{eff}}\left[\mathbf{r}_{i}-\mathbf{r}_{j}^{k}(t)\right] \\
& \left.+V_{\mathrm{ext}}\left(\mathbf{r}_{i}, t\right)\right] \varphi_{i}^{k}\left(\mathbf{r}_{i}, t\right),
\end{aligned}
$$

where the effective electron-electron potential $V_{e-e}^{\mathrm{eff}}\left[\mathbf{r}_{i}-\mathbf{r}_{j}^{k}(t)\right]$ is expressed as a Monte Carlo sum over the smoothed walker distribution [9]:

$$
\begin{aligned}
& V_{e-e}^{\mathrm{eff}}\left[\mathbf{r}_{i}-\mathbf{r}_{j}^{k}(t)\right] \\
& \quad=\frac{1}{Z_{j}^{k}} \sum_{l=1}^{M} V_{e-e}^{\mathrm{scr}}\left[\mathbf{r}_{i}-\mathbf{r}_{j}^{l}(t)\right] K\left(\frac{\left|\mathbf{r}_{j}^{l}(t)-\mathbf{r}_{j}^{k}(t)\right|}{\sigma_{j}^{k}\left(\mathbf{r}_{j}^{k}, t\right)}\right),
\end{aligned}
$$

where

$$
Z_{j}^{k}=\sum_{l=1}^{M} K\left(\frac{\left|\mathbf{r}_{j}^{l}(t)-\mathbf{r}_{j}^{k}(t)\right|}{\sigma_{j}^{k}\left(\mathbf{r}_{j}^{k}, t\right)}\right)
$$

where $K$ is a smoothing kernel and $Z_{j}^{k}$ is the weighting factor. The width $\sigma_{j}^{k}\left(\mathbf{r}_{j}^{k}, t\right)$ of the kernel in (7) is a measure for the characteristic length of nonlocal quantum correlations within the ensemble of walkers which represent the $j$ th electron. In practice, the parameter $\sigma_{j}^{k}\left(\mathbf{r}_{j}^{k}, t\right)$ is determined by variationally minimizing the ground state energy of the quantum system [13].

In our calculation a Coulomb potential screened by an error function is used [10]:

$$
V_{e-e}^{\mathrm{scr}}\left[\mathbf{r}_{i}-\mathbf{r}_{j}^{l}(t)\right]=V_{e-e}\left[\mathbf{r}_{i}-\mathbf{r}_{j}^{l}(t)\right] \operatorname{erf}\left[\frac{\left|\mathbf{r}_{i}-\mathbf{r}_{j}^{l}(t)\right|}{r_{j}^{s} \delta_{s i, s j}}\right],
$$

where the Kronecker symbol $\delta_{s i, s j}$ restricts the screening effect to the repulsion between only the same-spin walkers, 
while the value of screening parameter $r_{i}^{s}$ is estimated from the Hartree-Fock approximation.

In the approach outlined previously, a self-interactionfree dynamics in physical space is achieved, where the separate walkers do not share guiding waves which represent different distributions. In order to calculate the many-body probability distribution in configuration space, a separate auxiliary set of walkers with primed coordinates $\mathbf{r}_{i}^{\prime k}$ is introduced which is guided by an antisymmetric wave function:

$$
\begin{aligned}
& \mathbf{v}^{\prime}\left(\mathbf{r}_{i}^{\prime k}\right) \\
& \quad=\frac{\hbar}{m} \operatorname{Im}\left[\frac{1}{\Psi^{\prime k}\left(\mathbf{r}_{1}^{\prime}, \ldots, \mathbf{r}_{N}^{\prime}, t\right)} \nabla_{i} \Psi^{\prime k}\left(\mathbf{r}_{1}^{\prime}, \ldots, \mathbf{r}_{N}^{\prime}, t\right)\right]_{\mathbf{r}_{j}^{\prime}=\mathbf{r}_{j}^{\prime k}(t)},
\end{aligned}
$$

where $\Psi^{\prime k}\left(\mathbf{r}_{1}^{\prime}, \ldots, \mathbf{r}_{N}^{\prime}, t\right)$ is an antisymmetrized product (Slater determinant or a sum of Slater determinants) of the timedependent guide waves $\varphi_{i}^{k}\left(\mathbf{r}_{i}, t\right)$ of (6):

$$
\Psi^{\prime k}\left(\mathbf{r}_{1}^{\prime}, \mathbf{r}_{2}^{\prime}, \ldots, \mathbf{r}_{N}^{\prime}, t\right)=A \prod_{i=1}^{N} \varphi_{i}^{k}\left(\mathbf{r}_{i}^{\prime}, t\right)
$$

From (10) and (11) one can see that each walker with primed coordinates samples the many-body wave function, and thus it belongs to all guide waves (i.e., it represents an indistinguishable electron). The distribution of these walkers can be used to directly estimate the average in (1) by reducing it to (for states with spherical symmetry)

$$
I(u, t) \propto \sum_{i} K_{i}\left[\frac{\left|r_{12}^{\prime i}(t)-u\right|}{\sigma_{12}^{i}}\right],
$$

where $r_{12}^{\prime i}(t)=\left|\mathbf{r}_{1}^{\prime i}(t)-\mathbf{r}_{2}^{\prime i}(t)\right|$. In other words, the pair-density function can be simplified to a smoothed histogram (or a kernel density estimation with kernel $K_{i}$ and bandwidth $\sigma_{12}^{i}$ [14]) over the ensemble of the distances between the primed walkers.

\section{Exchange and Coulomb Correlations in Helium}

The two major sources of electron-electron correlation are due to the symmetry of the quantum state and due to the Coulomb repulsion. Here we consider first the effect of the exchange correlation on the pair-density function of helium atom. Although the electron-pair densities for helium have been analyzed by different techniques, they have never, to the author's knowledge, been studied using time-dependent methods.

In order to examine the electron correlation which is due to the exchange interaction, we consider the spin-triplet ground state of helium (orthohelium). The preparation of the ground state is described elsewhere [11,12]. In the calculation here we use up to 100000 Monte Carlo walkers and the same number of guiding waves, which are propagated over 2000 complex time steps (see (5) through (10)) in the presence

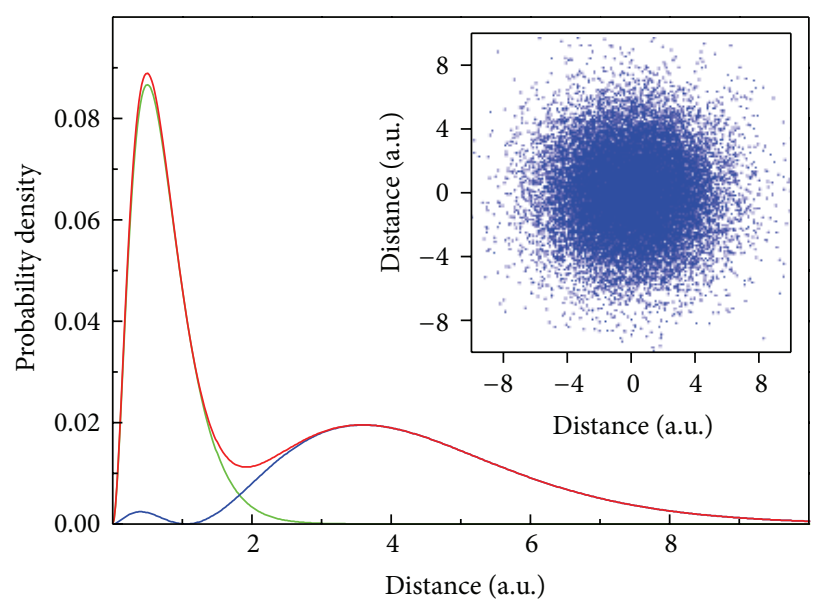

FIGURE 1: Radial electron density for the ground state of orthohelium, for MC walkers guided in physical space (blue and green lines), and for MC walkers guided in configuration space (red line). The inset shows the projection of the coordinates of the MC walkers in the $x-y$ plane.

of random component in walker's motion such that each walker samples the distribution given by its own guiding wave. In order to determine the screening parameter $r_{i}^{s}$ of (9), we invoke the Hartree-Fock approximation, where for $\sigma_{j}^{k}\left(\mathbf{r}_{j}^{k}, t\right) \rightarrow \infty$ the Coulomb potential in (7) reduces to a simple (unweighted) sum of the Coulomb potentials due to all walkers. Because of the spherical symmetry of the $2{ }^{3} \mathrm{~S}$ state $r_{i}^{s}$ is being varied until minimizing the mean integrated squared error of the walker's distribution against the probability distribution obtained from an independent HartreeFock solution (e.g., in [15]). Figure 1 shows the probability distributions obtained from TDQMC for the optimizing value of $r_{i}^{s}=r^{s}=1.13$ a.u. in (9). The blue and the green lines show the densities of the walkers guided in physical space (see (5) through (9)), respectively, while the red line represents the radial distribution of the walkers guided in configuration space (see (10)). In these calculations a new accurate algorithm for kernel density estimation was used [16]. Notice that all probability distributions throughout this paper are normalized to unity.

The electron-pair density for the ground state was calculated very efficiently by simply performing kernel density estimation over the ensemble of distances between the primed walkers. The result is shown in Figure 2(a) where the blue and the red lines present the cases with and without exchange interaction, respectively. The lack of exchange $\left(r_{i}^{s} \rightarrow 0\right.$ in (9)) leads to a full (unscreened) Coulomb repulsion, which in the limit of infinite nonlocal correlation length $\left(\sigma_{j}^{k}\left(\mathbf{r}_{j}^{k}, t\right) \rightarrow \infty\right)$ becomes equivalent to the Hartree approximation. Figure 2(b) shows the difference between the two curves in Figure 2(a), which in fact depicts the shape of the exchange hole for the $2{ }^{3} \mathrm{~S}$ state of helium (see also e.g., [5]). Note that the exchange hole in our calculation may differ from other results because the distribution of the Monte Carlo walkers varies in radial direction as $r^{2} R^{2}(r)$ 


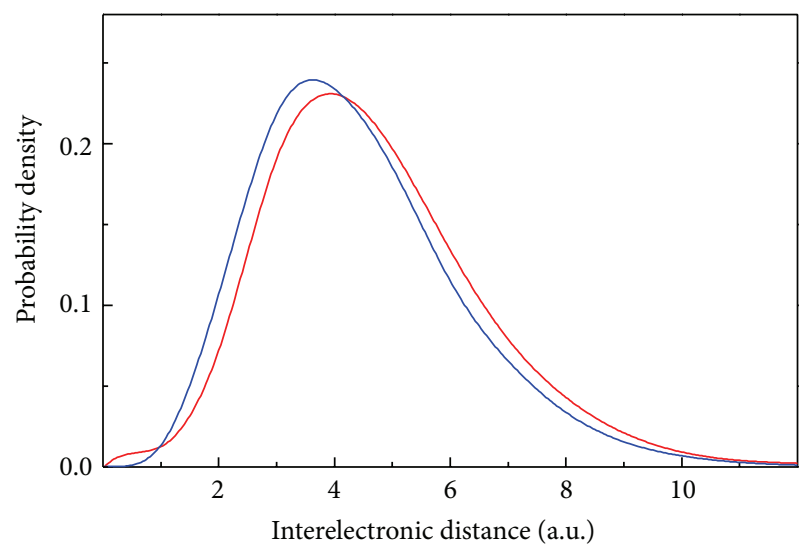

(a)

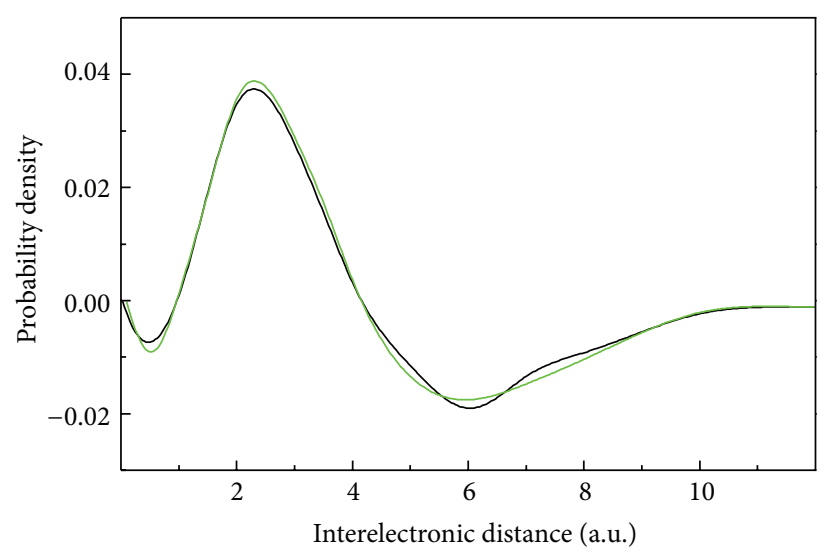

(b)

Figure 2: Electron-pair density as function of the interelectronic distance, for the ground state of orthohelium. (a) Red line-no screening (no exchange), blue line-short-range screened Coulomb potentials. Exchange hole (b) for screened Coulomb potentials (black) and for Hartree-Fock exchange (green).

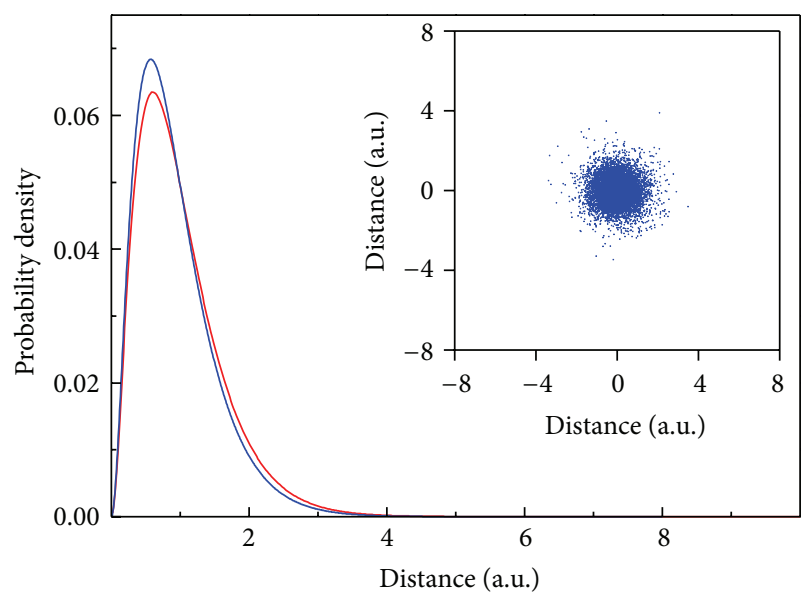

FIGURE 3: Radial electron density for the ground state of parahelium, for MC walkers guided in physical space (red line), and from the Hartree-Fock approximation (blue line). The inset shows the projection of the coordinates of the MC walkers in the $x-y$ plane.

instead of as $R^{2}(r)$, where $R(r)$ is the radial wave function. The green line in Figure 2(b) shows the exchange hole obtained from an independent Hartree-Fock calculation with no potential screening. It is seen that the two curves are close where the deviations for larger interelectronic distances are mainly due to the fast decrease of the walker's density away from the core. As the screening parameter $r_{i}^{s}$ tends to zero both the height and the width of the exchange hole decrease until the two curves in Figure 2(b) become very close, with the only remaining difference being a result of purely Coulomb correlations.

For the ground state of the $2{ }^{1} \mathrm{~S}$ (para)helium, the quantity of interest is the Coulomb hole which occurs due to the repulsion of the closely spaced walkers. Figure 3 shows the probability distribution of the ground state walkers as compared to the Hartree-Fock calculation, while Figure 4(a) depicts the corresponding interelectronic distances for the two cases. The Coulomb hole calculated as the difference between the two curves is presented in Figure 4(b) which is close to previous results by other methods [3]. As the nonlocal correlation length $\sigma_{j}^{k}\left(\mathbf{r}_{j}^{k}, t\right)$ tends to infinity, both the height and the width of the Coulomb hole decrease until the two curves in Figure 4(b) coincide. Thus, in our approach where the exchange and the Coulomb correlations are accounted for by solely modifying the potential of electron-electron interaction in physical space, the two parameters $r_{i}^{s}$ and $\sigma_{j}^{k}\left(\mathbf{r}_{j}^{k}, t\right)$ may ensure a smooth transition between the Hartree, the Hartree-Fock, and the fully correlated approximations to the electron-electron interaction. It is important to point out that in the $\hbar / m \rightarrow 0$ limit the quantum drift in (6) vanishes and so does the width of the quantum wave packet. Therefore, for an isolated atom the quantum correlation length $\sigma_{j}^{k}\left(\mathbf{r}_{j}^{k}, t\right)$ tends to zero in this limit, and if there are no exchange effects $\left(r_{i}^{s} \rightarrow 0\right)$, the ensemble of quantum particles governed by (5) and (6) transforms to an ensemble of classical particles with the only force being due to the standard Coulomb repulsion between these particles.

\section{Conclusions}

In this paper, it has been shown that for charged particles, the quantum correlation effects which occur due to the exchange and Coulomb correlations can adequately be described by sets of de Broglie-Bohm walkers within the time-dependent quantum Monte Carlo framework. A short-range screening of the Coulomb potential ensures that each replica of a given electron interacts with only those replicas of the rest of the same spin electrons which are sufficiently apart to respect Pauli's exclusion principle in space. On the other hand, the electron-electron interaction is modified by the quantum nonlocality which demands that each replica of a given electron interacts with the replicas of the other electrons which are within the range of the nonlocal quantum correlation length. This concept allows one to build a robust, self-consistent, and self-interaction-free approach to find 


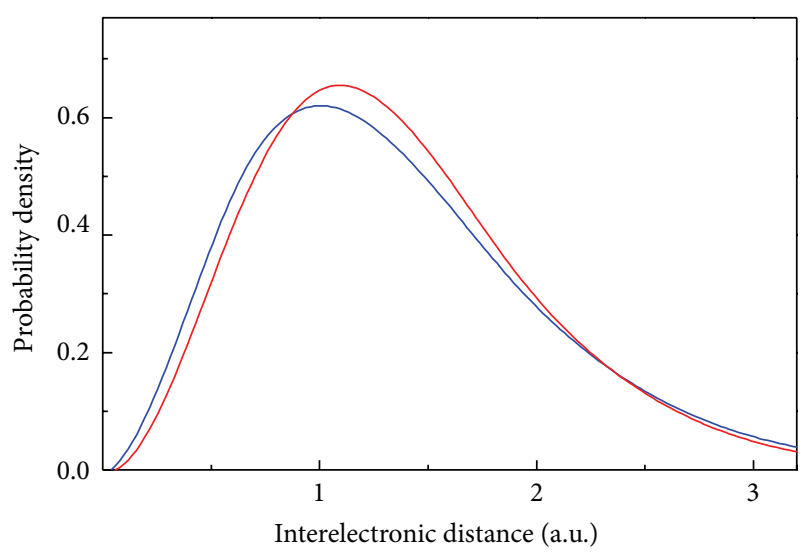

(a)

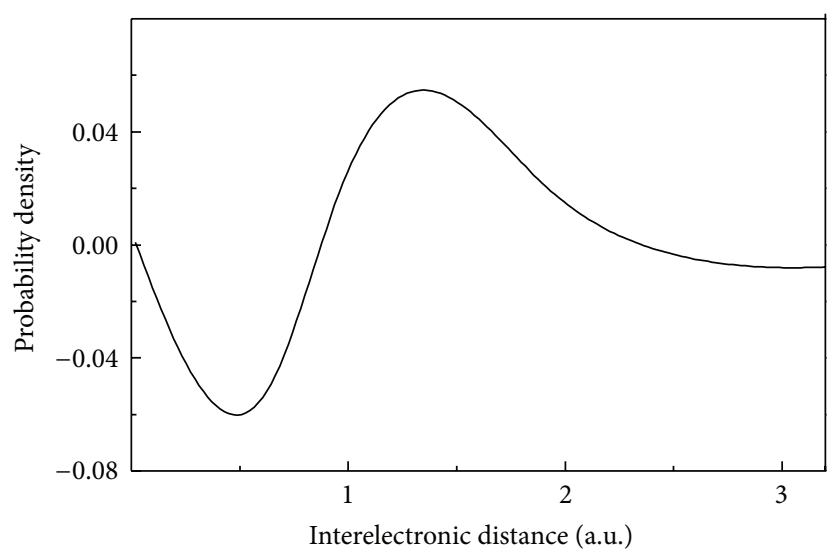

(b)

FIGURE 4: Electron-pair density as function of the interelectronic distance for the ground state of parahelium. (a) Red line-correlated result, blue line-Hartree-Fock approximation. The Coulomb hole (b).

both the ground state and the time evolution of quantum systems. It is demonstrated here that the otherwise awkward procedure for calculating the pair distribution functions of para- and orthohelium atom can be simplified to the level of finding the ground state probability distributions of the corresponding Monte Carlo walkers.

Besides the relative ease of its implementation, another advantage of using TDQMC is the affordable time scaling it offers which is almost linear with the system dimensionality. This is especially valid when using multicore parallel computers where little communication overhead between the different processes can be achieved, thus utilizing the inherent parallelism of the Monte Carlo methods. This nears the TDQMC to other efficient procedures for treating manybody quantum dynamics such as the time-dependent density functional approximation which, however, suffers systematic self-interaction problems due to the semiempirical character of the exchange-correlation potentials.

\section{Acknowledgments}

The author gratefully acknowledges support from the National Science Fund of Bulgaria under Grant DCVP 02/1 (SuperCA++). Computational resources from the National Supercomputer Center (Sofia) are gratefully appreciated.

\section{References}

[1] A. J. Coleman, "Density matrices in the quantum theory of matter: energy, intracules and extracules," International Journal of Quantum Chemistry, vol. 1, supplement 1, pp. 457-464, 1967.

[2] A. J. Thakkar, "Extracules, intracules, correlation holes, potentials, coefficients and all that," in Density Matrices and Density Functionals, R. Erdahl and V. H. Smith Jr, Eds., pp. 553-581, Reidel, New York, NY, USA, 1987.

[3] C. A. Coulson and A. H. Neilson, "Electron correlation in the ground state of helium," Proceedings of the Physical Society, vol. 78, no. 5, p. 831, 1961.

[4] R. J. Boyd and C. A. Coulson, "The Fermi hole in atoms," Journal of Physics B, vol. 7, no. 14, pp. 1805-1816, 1974.
[5] N. Moiseyev, J. Katriel, and R. J. Boyd, "On the Fermi hole in atoms," Journal of Physics B, vol. 8, no. 8, pp. L130-L133, 1975.

[6] P. M. W. Gill, D. O’Neill, and N. A. Besley, "Two-electron distribution functions and intracules," Theoretical Chemistry Accounts, vol. 109, no. 5, pp. 241-250, 2003.

[7] B. M. Austin, D. Y. Zubarev, and W. A. Lester, "Quantum monte carlo and related approaches," Chemical Reviews, vol. 112, no. 1, pp. 263-288, 2012.

[8] I. P. Christov, "Correlated non-perturbative electron dynamics with quantum trajectories," Optics Express, vol. 14, no. 15, pp. 6906-6911, 2006.

[9] I. P. Christov, "Dynamic correlations with time-dependent quantum Monte Carlo," Journal of Chemical Physics, vol. 128, no. 24, Article ID 244106, 2008.

[10] I. P. Christov, "Polynomial-time-scaling quantum dynamics with time-dependent quantum Monte Carlo," The Journal of Physical Chemistry A, vol. 113, pp. 6016-6021, 2009.

[11] I. P. Christov, "Correlated electron dynamics with time-dependent quantum Monte Carlo: three-dimensional helium," Journal of Chemical Physics, vol. 135, no. 4, Article ID 044120, 2011.

[12] I. P. Christov, "Erratum "Correlated electron dynamics with time-dependent quantum Monte Carlo: three-dimensional helium'”' Journal of Chemical Physics, vol. 135, no. 14, Article ID 149902, 2011.

[13] I. P. Christov, "Exploring quantum non-locality with de BroglieBohm trajectories," Journal of Chemical Physics, vol. 136, no. 3, Article ID 034116, 2012.

[14] B. W. Silverman, Density Estimation for Statistics and Data Analysis, Monographs on Statistics and Applied Probability, Chapman and Hall, London, UK, 1986.

[15] S. E. Koonin and D. C. Meredith, Computational Physics, Addison-Wesley, 1990.

[16] Z. I. Botev, J. F. Grotowski, and D. P. Kroese, "Kernel density estimation via diffusion," The Annals of Statistics, vol. 38, no. 5, pp. 2916-2957, 2010. 

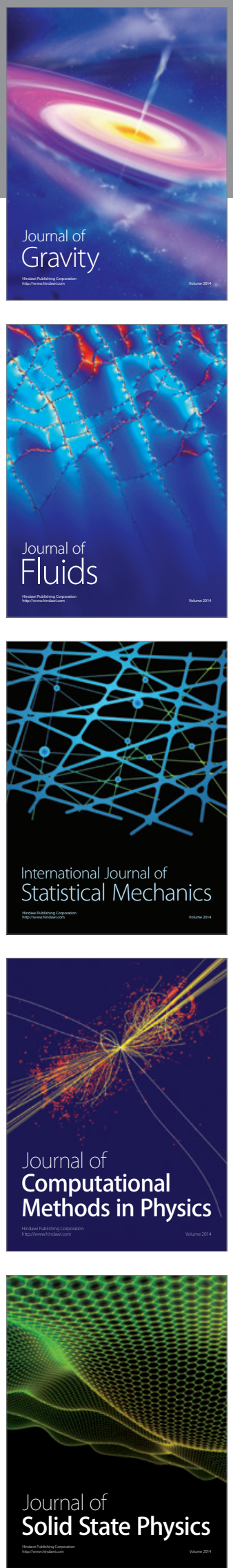

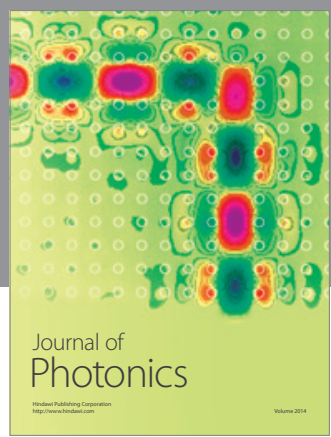

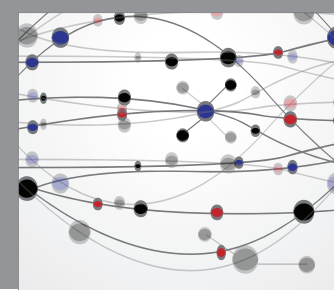

The Scientific World Journal

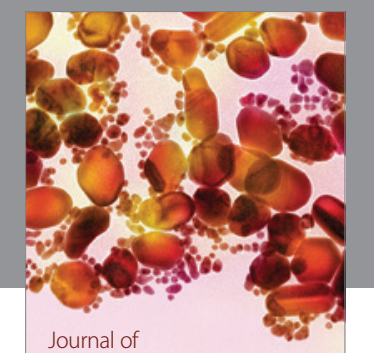

Soft Matter
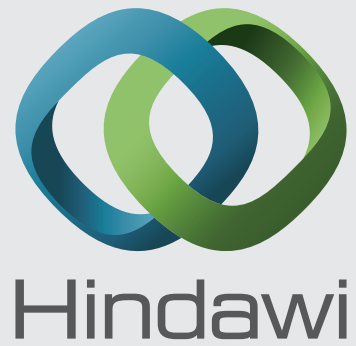

Submit your manuscripts at

http://www.hindawi.com
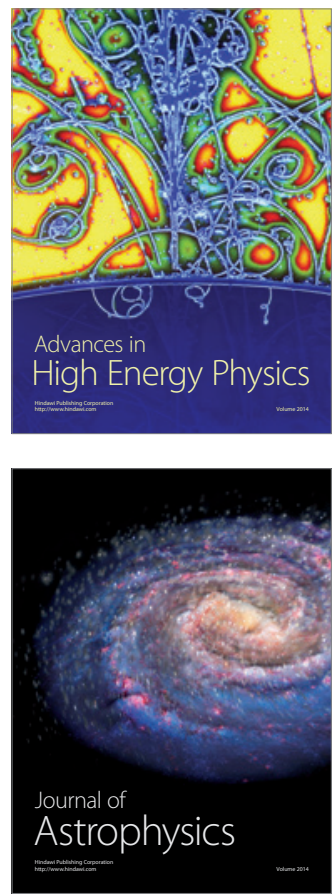
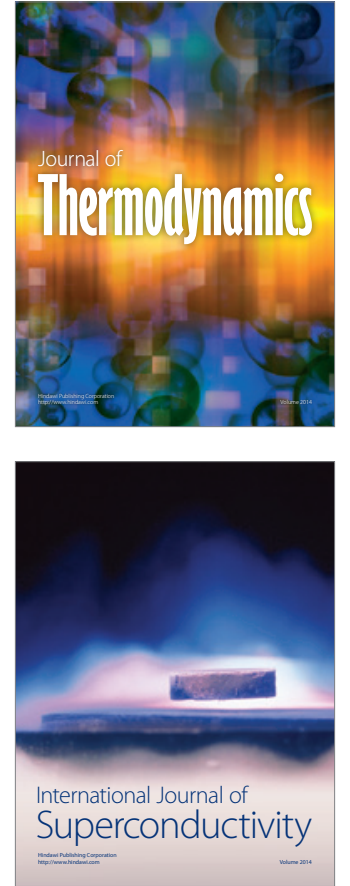
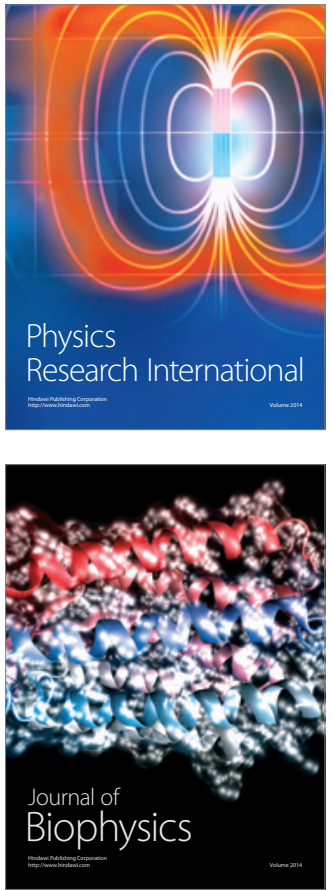
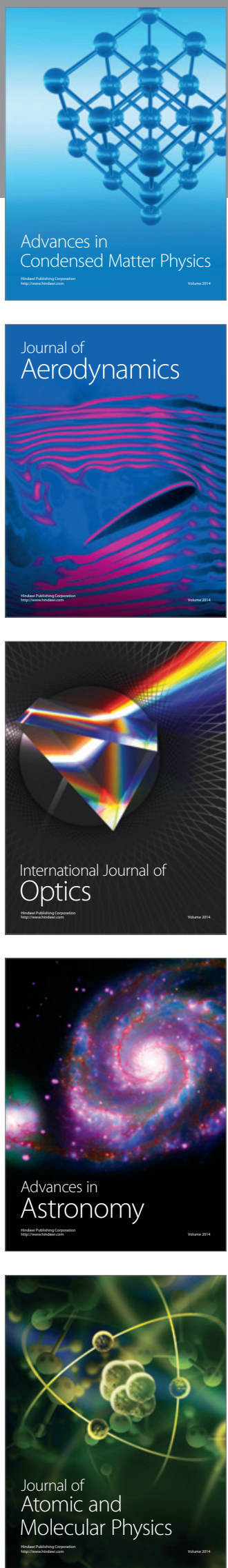